\title{
Effect of Exercise on Fatigue in Multiple Sclerosis Patients: A Network Meta-analysis
}

\author{
Authors \\ Affiliations \\ 1 Department of Epidemiology and Biostatistics, Anhui \\ Medical University, Hefei, Anhui, China \\ 2 Department of Internal Medicine, First Affiliated Hospital \\ of Anhui Medical University, Hefei, Anhui, China \\ 3 The Key Laboratory of Major Autoimmune Diseases, \\ Anhui Medical University, Hefei, Anhui, China
}

Yuting Chen ${ }^{\# 1,3}$, Shanshan $\mathrm{Xu}^{\# 1,3}$, Jiran Shen ${ }^{2,3}$, Hui Yang ${ }^{1,3}$, Wei $\mathrm{Xu}^{1,3}$, Ming Shao1, , Faming Pan ${ }^{1,3}$

Key words

multiple sclerosis, fatigue, exercise, training, ranking

accepted 11.05 .2021

published online $\quad 10.08 .2021$

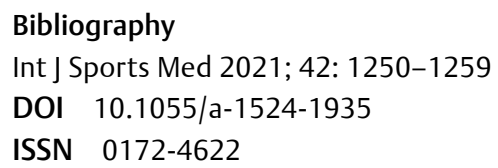

(c) 2021. Thieme. All rights reserved. Georg Thieme Verlag, Rüdigerstraße 14, 70469 Stuttgart, Germany

\section{Correspondence}

Faming Pan

Department of Epidemiology and Biostatistics

Anhui Medical University

81 Meishan Road

Hefei

Anhui

230032

China

Tel.: 15856954540, Fax: 15856954540

famingpan@ahmu.edu.cn $\circledast \begin{aligned} & \text { Supplementary material is available under } \\ & \text { https://doi.org/10.1055/a-1524-1935 }\end{aligned}$

\begin{abstract}
Few studies have directly compared the effects of different exercise therapies on reducing fatigue in patients with multiple sclerosis. Thus, we conducted a Frequentist network metaanalysis to analyze and compare the effectiveness of different types of exercise on reducing multiple sclerosis-related fatigue. Relevant randomized controlled trials were searched in PubMed, Web of Science and Cochrane Library databases from the date of their inception up to April 1, 2021. In total, 27 articles involving 1470 participants and 10 types of interventions met the inclusion criteria. The results indicated that aquatic exercise ranked as the most effective among these interventions, and aerobic exercise had small-to-moderate effect sizes. Most of the interventions were shown to be better than the control group, except for climbing. Climbing was the only intervention that ranked worse than the controls. All of these findings merit further investigation in future clinical trials.
\end{abstract}

\section{Introduction}

Multiple sclerosis (MS) is often described as a chronic, inflammatory disorder of the central nervous system that is characterized by demyelination and axonal loss [1]. MS is one of the leading causes of non-traumatic disability in young adults, and there are more than 2.3 million people with MS worldwide [2]. The global median prevalence of MS is 33 per 100000 population, with significant dif-

\# Yuting Chen and Shanshan Xu contributed equally to this work and should be considered as main contributors. ferences between countries [3]. Common manifestations in MS include, but are not limited to, symptomatic fatigue, depression, spasticity, mobility and balance problems, cognitive decline, and muscle weakness [4-9]. Among them, fatigue is intrinsic to MS and is the most frequently reported symptom [10]. Up to $75-90 \%$ of people with MS complain of fatigue, and $60 \%$ regard it as the most disabling complication that seriously reduces quality of life [11]. People with MS tend to engage in less physical activity than the general population, which in turn increases fatigue and the risk of developing secondary diseases such as obesity and diabetes 
$[12,13]$. The bi-directional connections between fatigue and physical inactivity accelerate the functional decline in patients with MS.

For years, people with MS were advised not to take part in exercise because it was thought to cause worsening symptoms or fatigue. However, in recent decades, a number of types of exercise have been shown to reduce MS-related fatigue in extensive trials, systematic reviews, and meta-analyses, such as aerobic exercise, yoga, resistance training, and endurance training [14-16]. The American Physical Therapy Association has built preferred practice patterns for patients, including those with MS [17]. However, information about specific patterns of physical activity in MS patients is still limited. Previous meta-analyses reported that exercise could reduce fatigue in MS patients, but all of these studies were traditional pairwise meta-analyses that only considered direct comparisons and did not rank the interventions [18-20]. It remains unclear which anti-fatigue intervention has the greatest effects on relieving fatigue. Therefore, it is a problem for MS patients to find the most effective anti-fatigue interventions based on their interests and needs.

To provide a comprehensive overview, we applied the network meta-analysis (NMA) approach to analyze and compare the effectiveness of different types of exercise on relieving MS-related fatigue.

\section{Materials and Methods}

The study was performed in line with the guidelines from the Preferred Reporting Items for Systematic Reviews and Meta-Analyses and the Cochrane Intervention Review that Compares Multiple Interventions, as well as the Ethical Standards in Sport and Exercise Science Research and this journal [21, 22]. The protocol was previously written but was not registered.

In this NMA, aerobic exercise is defined as a unique form of activity, which involves the integration of large muscle groups, such as the rhythmic propulsion of body mass during the movements of varying intensities (for example, walking, jogging, or running) or activities with lower mechanical impact (for example, cycling) [23]. Resistance training is a type of progressive overload strength training in which the muscles exert force against an external load [24]. Endurance training is characterized by the repeated isotonic contraction of large skeletal muscle groups (for example, cross-country skiing or speed skating in winter sports) [25]. Aquatic exercise, also known as pool therapy, hydrotherapy, or balneotherapy, mainly describes exercise that is done in the water.

\section{Search strategy and selection criteria}

PubMed, Web of Science, Cochrane Library of Systematic Reviews and Cochrane Controlled Clinical Trials databases were searched from the date of their inception up to April 1, 2021 to collect relevant randomized controlled trials (RCTs). Keywords and search strategy were as follows: ("multiple sclerosis" OR “MS") AND ("fatigue” OR “lassitude”) AND (random *). Titles, abstracts, and key words were scanned, and full-text articles were evaluated if they met the inclusion criteria. The references of the included studies, systematic reviews, and meta-analyses were also manually searched to find additional topic-related literature. In the case of insufficient data, we tried to email the corresponding author to obtain the necessary information.
A number of studies were retrieved, but only studies meeting the following criteria were considered eligible: (1) an RCT protocol was applied to evaluate the effect of at least one exercise arm; (2) participants were adults who were clinically diagnosed with MS; (3) a questionnaire was used at baseline and follow-up to evaluate fatigue symptoms; (4) sufficient data should have been provided to calculate the effect sizes of outcome variables; and (5) studies were published in English.

\section{Exclusion criteria}

Our study focused on different types of exercise on alleviating fatigue in MS patients. Therefore, trials comparing drugs, neurofeedback, acupuncture, electroacupuncture, acupressure, energy conservation management, light therapy, and other contactless rehabilitation (e.g., telephone-administered healing) were excluded. In addition, studies with intervention periods $<2$ weeks were excluded. Quasi-RCTs, conference abstracts, editorials, letters, and case reports were also excluded.

\section{Data extraction}

Two reviewers independently extracted data using a standardized scheme. Extracted data were double-checked by the corresponding author. Any discrepancies were resolved by discussion and careful reexamination until an agreement was reached. The following information was collected: first author's name, publication year, country, sample size, sex ratio, age, characteristics of participants, type of intervention, duration of intervention, outcome measurements, and other additional information.

\section{Risk of bias and quality assessment}

Risk of bias in the included studies was assessed by using Cochrane Collaboration's Tool for Assessing Risk of Bias [26]. We evaluated the studies based on seven criteria (random sequence generation, allocation concealment, blinding of participants and personnel, blinding of outcome assessment, incomplete outcome data, selective reporting, and other sources of bias). Each study was scored as high, low, and unclear risk of bias. The process was completed by two reviewers independently, and consensus was reached by consulting another author.

\section{Summary of outcomes}

Our primary outcome variables included mean, standard deviations (SD), and sample sizes ( $n$ ) to calculate the mean change from baseline to endpoint in fatigue symptoms. If more than one post-treatment fatigue score was reported in a study, we used only the pointin-time score that was assessed immediately after the end of the intervention period. When data were presented as median, range and/or interquartile range, we transformed them to mean and SD [27]. Fatigue was measured by different assessment scales, and the standard mean difference (SMD) was used as the effect size index to eliminate the influence of metrics. SMD values of $0.2,0.5$, and 0.8 indicate small, medium, and large effect sizes, respectively [28].

\section{Data synthesis}

An NMA combining direct and indirect evidence in a network of trials was conducted, and a random effects model was adopted to compare the relative effects of each intervention [29]. All data anal- 
ysis and graph generation were completed by using Stata 11.0 statistical software (StataCorp, College Station, TX, USA).

First, a network plot visually expressed the comparison between different interventions. Moreover, a forest plot showed the SMD based on sample sizes and their $95 \%$ confidence interval $(\mathrm{Cl})$, and heterogeneity evaluated by Higgins ${ }^{2}$ was also given. Additionally, surface under the cumulative ranking curve (SUCRA) probabilities were calculated using the Markov chain Monte Carlo (MCMC) model to rank the interventions [30]. The SUCRA indicated the relative probability of each studied intervention among the optimal options, and a larger SUCRA value represents a higher ranking of an intervention [31]. A two-sided $p<0.05$ was considered as statistically significant. The application of the network meta-analysis method overcame the lack of direct comparisons and allowed for a comprehensive conclusion.

\section{Assessment of inconsistency}

The loop-specific model and Wald test were used to examine the global consistency between direct and indirect comparisons $[32,33]$. The $95 \% \mathrm{Cl}$ of inconsistency factor (IF) excludes 0 or the alpha value $\leq 0.05$ indicating statistically significant inconsistency. The node-splitting model was adopted to further explore the local inconsistency across each network.

\section{Subgroup analysis and sensitive analysis}

To examine the sources of heterogeneity, subgroup analysis was performed according to median intervention duration and median age. Because of the characteristics of exercise therapy, it is impossible to blind both participants and personnel. The included studies tend to have a high blinding risk. Therefore, sensitive analysis was performed by excluding studies that failed to achieve outcome assessment blinding.

\section{Meta-regression analysis and publication bias}

Meta-regression analysis was performed by publication year, sex ratio, sample size and intention to treat. Additionally, a funnel plot was used to detect potential publication bias and small-study effects.

\section{Results}

\section{Study identification and selection}

A total of 4252 records were identified in the online databases listed above, 958 from PubMed, 1,346 from Web of Science, and 1,948 from Cochrane Library, respectively. After removal of the duplicates, titles and abstracts of 2524 records were browsed to find potential relevance. A total of 182 articles was included in the full-text assessment. Finally, 27 articles satisfied the inclusion criteria and were considered as eligible for further analyses. The flowchart of the whole literature retrieval process is shown in > Fig. 1 .

\section{Characteristics of the studies}

The baseline characteristics of the studies included in this NMA are detailed in > Table 1. All 27 articles were published in English between 1996 and 2019. Sample sizes ranged from 11 to 314 patients; 6 came from Iran [34-39], 4 from America [40-43]; 3 each from Britain [44-46] and Germany [47-49]; two each from Italy $[50,51]$, Denmark [52, 53], and Australia [54, 55]; and 1 each from

4252 records identified through PubMed $(n=958)$,

Web of Science $(n=1346)$,

Cochrane CENTRAL $(n=1948)$

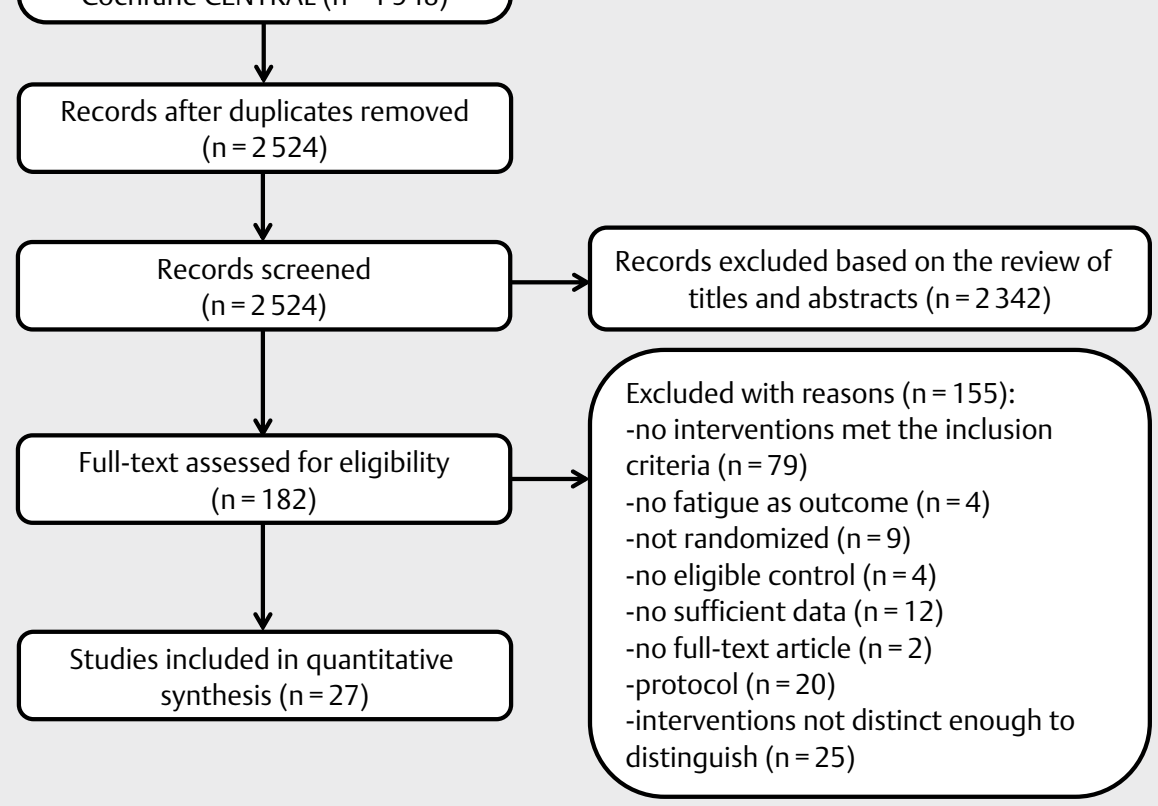

Fig. 1 Flow chart of the study selection process. 


\begin{tabular}{|c|c|c|c|c|c|c|c|c|c|c|c|c|c|c|c|}
\hline 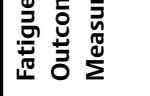 & $\tilde{y}$ & $\tilde{\mu}$ & 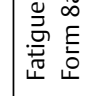 & $\tilde{\omega}$ & 竞 & | & 接 & 㝈 & $\frac{\frac{\omega}{\frac{1}{2}}}{2}$ & 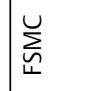 & $\tilde{\omega}$ & 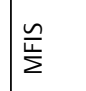 & $\tilde{\omega}$ & $\frac{\omega}{\frac{\omega}{2}}$ & $\frac{u}{\frac{u}{2}}$ \\
\hline 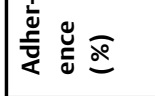 & $\cong$ & $\frac{\alpha}{z}$ & 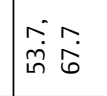 & 苟 & I & \pm & $\frac{\alpha}{z}$ & $\frac{\alpha}{z}$ & 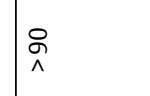 & $\%$ & $\frac{\alpha}{z}$ & $\bar{\infty}$ & $\frac{\alpha}{z}$ & m & $\approx$ \\
\hline 晜离 & 厗 & 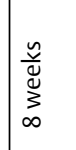 & 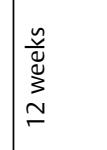 & 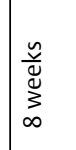 & 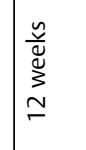 & 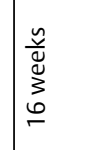 & 竞 & 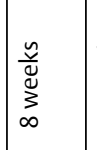 & 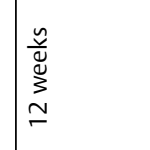 & 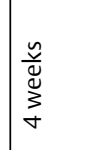 & 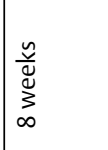 & 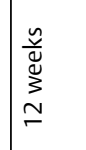 & 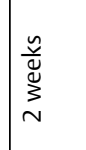 & 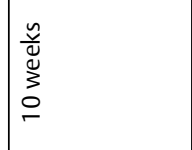 & 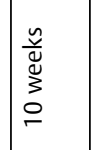 \\
\hline 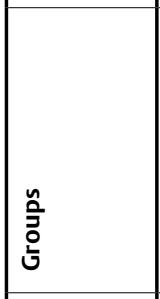 & 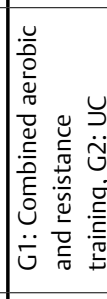 & & 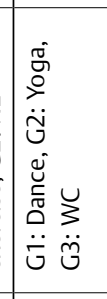 & 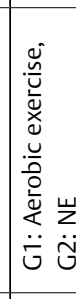 & 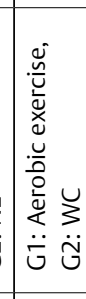 & 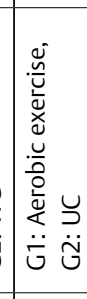 & 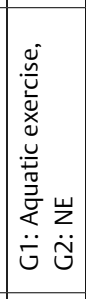 & 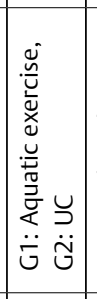 & 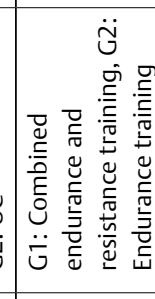 & 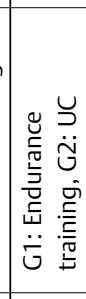 & 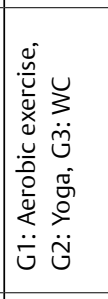 & 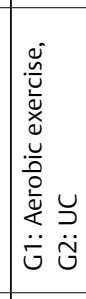 & 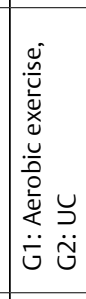 & 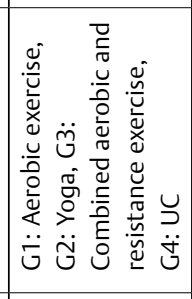 & 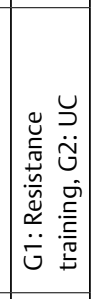 \\
\hline 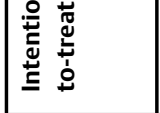 & $\frac{\alpha}{z}$ & $\frac{\alpha}{z}$ & $\frac{\alpha}{z}$ & $\frac{\alpha}{z}$ & $\stackrel{\sharp}{\longleftarrow}$ & $\stackrel{\check{\varkappa}}{\longleftarrow}$ & $\stackrel{\varpi}{\%}$ & $\frac{\alpha}{\frac{\alpha}{z}}$ & 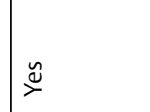 & $\frac{\rho}{z}$ & $\frac{\alpha}{z}$ & 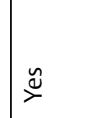 & $\frac{\alpha}{z}$ & $\frac{\alpha}{z}$ & 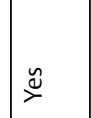 \\
\hline 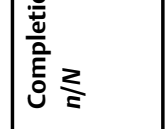 & $\frac{\partial}{\partial}$ & $\frac{\alpha}{\sigma}$ & $\frac{\bar{\alpha}}{\bar{\infty}}$ & $\frac{\frac{6}{6}}{\frac{6}{2}}$ & $\frac{7}{\frac{7}{m}}$ & 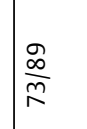 & $\mid \frac{\frac{8}{m}}{m}$ & $\mid \frac{o}{\frac{8}{m}}$ & $\frac{\frac{o}{m}}{m}$ & 产 & $\frac{\bar{m}}{\bar{m}}$ & 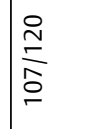 & 商 & $\mid \frac{\frac{d}{\frac{d}{d}}}{\frac{d}{d}}$ & $\frac{\mathscr{2}}{\hbar}$ \\
\hline 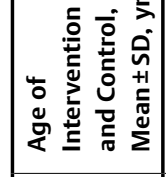 & 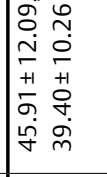 & 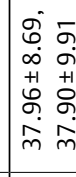 & 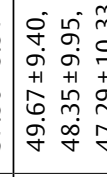 & 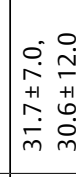 & 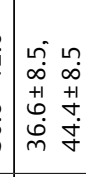 & 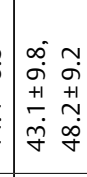 & & $\frac{\alpha}{z}$ & 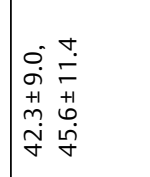 & 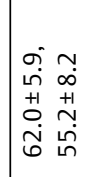 & 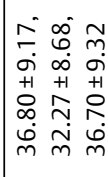 & 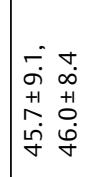 & 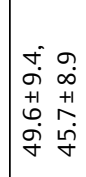 & 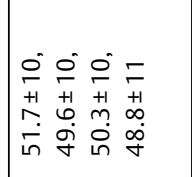 & 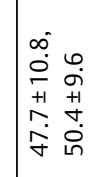 \\
\hline 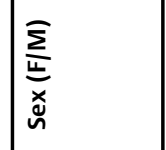 & $\begin{array}{l}n \\
\stackrel{n}{n} \\
\underline{n}\end{array}$ & $\begin{array}{l}0 \\
\dot{\sigma} \\
0\end{array}$ & 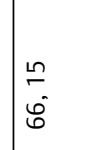 & $\mid \begin{array}{l}\bar{\alpha} \\
\bar{\sigma}\end{array}$ & $\mid \begin{array}{l}+ \\
\infty \\
\infty \\
m\end{array}$ & 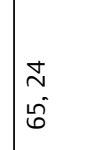 & $\mid \begin{array}{l}\dot{j} \\
\dot{m}\end{array}$ & $\begin{array}{l}0 \\
\dot{a}\end{array}$ & $\mid \frac{0}{7}$ & $\infty_{\infty}^{\infty}$ & $\frac{0}{\dot{m}}$ & 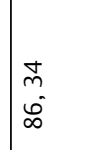 & $\frac{\infty}{\sim}$ & $\begin{array}{l}\mathbb{0} \\
\tilde{\infty} \\
\tilde{\infty}\end{array}$ & 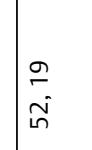 \\
\hline 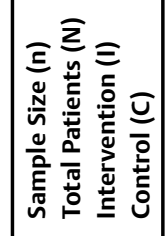 & 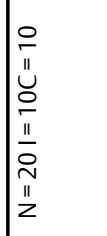 & 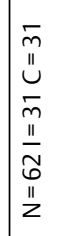 & 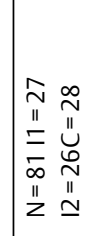 & 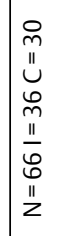 & $\begin{array}{l}\bar{\pi} \\
\pi \\
\tilde{\pi} \\
\frac{\pi}{2} \\
\pi \\
\frac{\pi}{2}\end{array}$ & 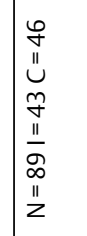 & 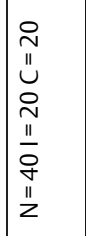 & 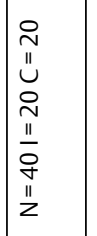 & 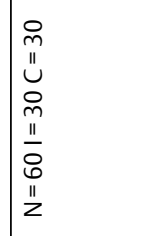 & $\mid \begin{array}{l}\frac{n}{3} \\
0 \\
\frac{i}{2} \\
\frac{\pi}{2} \\
\frac{\pi}{2}\end{array}$ & 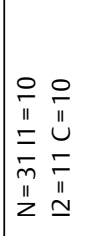 & $\begin{array}{l}0 \\
0 \\
0 \\
0 \\
0 \\
\frac{\pi}{2} \\
\frac{0}{\pi} \\
\frac{\pi}{2}\end{array}$ & 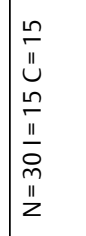 & 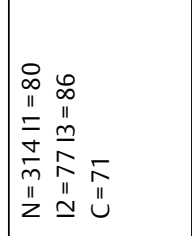 & 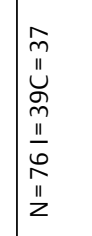 \\
\hline | & $\frac{8}{8}$ & 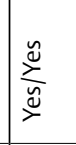 & 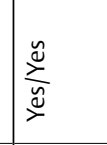 & 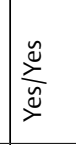 & 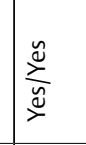 & 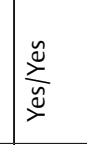 & $\frac{0}{\frac{0}{y}}$ & 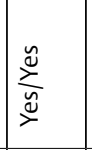 & 䓂 & 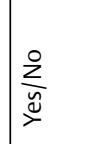 & 离 & 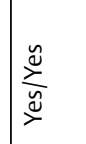 & 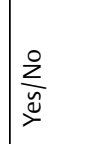 & 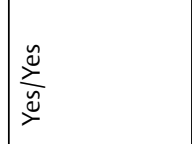 & 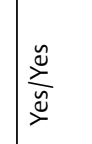 \\
\hline 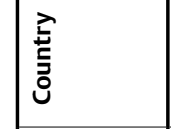 & 産 & $\underline{\underline{\underline{\underline{\underline{p}}}}}$ & $\begin{array}{l}\text { 莺 } \\
\text { 离 }\end{array}$ & $\underline{\underline{\underline{\underline{\underline{E}}}}}$ & 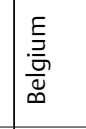 & 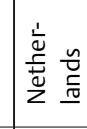 & 哥 & $\underline{\underline{\underline{\underline{E}}}}$ & 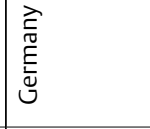 & \begin{tabular}{|l} 
蒿 \\
咅
\end{tabular} & $\underline{\underline{\underline{\underline{\underline{p}}}}}$ & 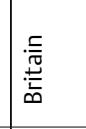 & 产 & 害䇏 & 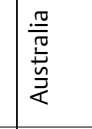 \\
\hline 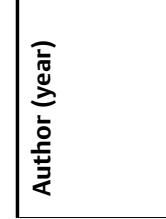 & 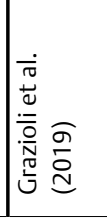 & & 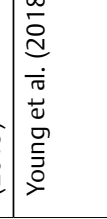 & & & 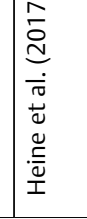 & & 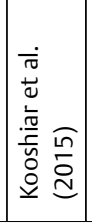 & 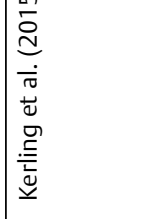 & & 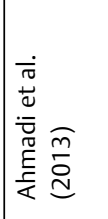 & 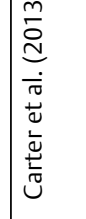 & & 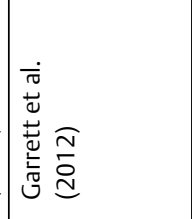 & 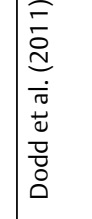 \\
\hline
\end{tabular}




\begin{tabular}{|c|c|c|c|c|c|c|c|c|c|c|c|c|c|}
\hline 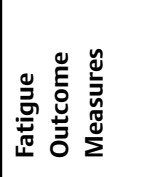 & $\frac{n}{\Sigma}$ & 岀 & $\tilde{\longleftarrow}$ & $\tilde{山}$ & $\frac{\tilde{n}}{\Sigma}$ & $\tilde{\iota}$ & $\frac{\frac{n}{u}}{\Sigma}$ & 豆 & $\frac{n}{\Sigma}$ & 苂 & 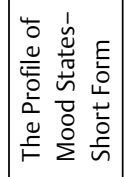 & $\widetilde{\varkappa}$ & 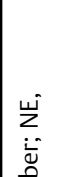 \\
\hline 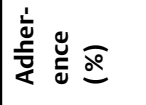 & $\frac{\alpha}{z}$ & $\frac{\propto}{z}$ & n̆ & $\frac{\alpha}{z}$ & $\frac{\varrho}{z}$ & $\frac{\varrho}{z}$ & $\frac{\alpha}{z}$ & $\frac{\alpha}{z}$ & $\frac{\alpha}{z}$ & $\frac{\alpha}{z}$ & $\frac{\alpha}{z}$ & à & $\begin{array}{l}\bar{E} \\
\bar{E} \\
z \\
\frac{\dot{\theta}}{n}\end{array}$ \\
\hline 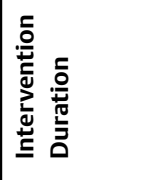 & 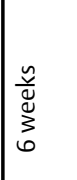 & 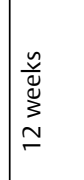 & 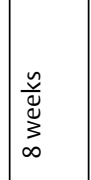 & 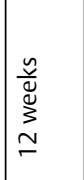 & 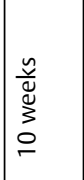 & 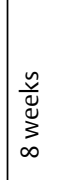 & 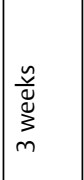 & 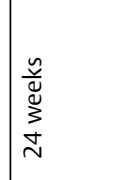 & 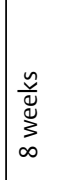 & 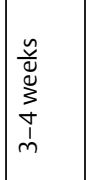 & 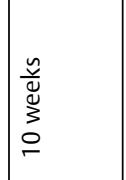 & 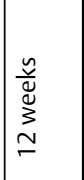 & 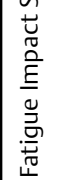 \\
\hline 参 & 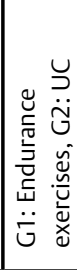 & 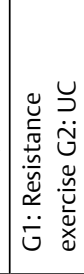 & 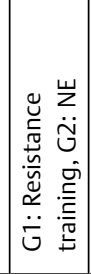 & 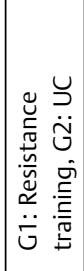 & 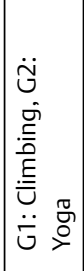 & 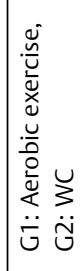 & 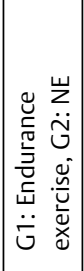 & 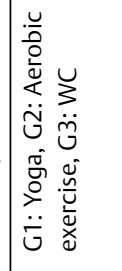 & 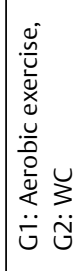 & 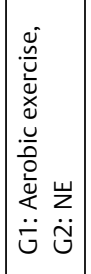 & 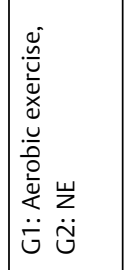 & 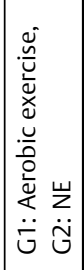 & 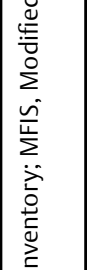 \\
\hline 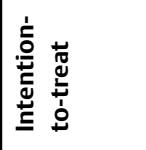 & $\stackrel{\check{\nu}}{\rightleftharpoons}$ & $\stackrel{\check{\varphi}}{\rightleftharpoons}$ & $\frac{\alpha}{z}$ & $\frac{\alpha}{z}$ & $\frac{\alpha}{z}$ & $\frac{\alpha}{z}$ & $\frac{\propto}{z}$ & $\frac{\alpha}{z}$ & $\frac{\varrho}{z}$ & $\frac{\alpha}{z}$ & $\frac{\alpha}{z}$ & $\frac{\alpha}{z}$ & 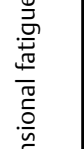 \\
\hline$\frac{5}{\frac{0}{0}}$ & $\frac{\stackrel{0}{N}}{\stackrel{N}{N}}$ & $\frac{\tilde{n}}{\stackrel{n}{N}}$ & $\frac{\stackrel{p}{m}}{N}$ & $\frac{\stackrel{\infty}{m}}{m}$ & $\frac{\stackrel{9}{\pi}}{\stackrel{N}{N}}$ & 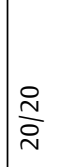 & $\frac{\bar{m}}{\mathrm{c}}$ & $\frac{8}{i n}$ & $\frac{\stackrel{\infty}{N}}{\stackrel{\infty}{\infty}}$ & 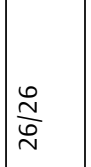 & $\frac{\underset{N}{N}}{\pi}$ & $\frac{g}{b}$ & 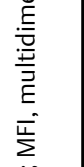 \\
\hline 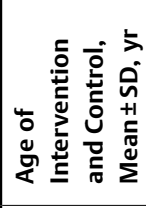 & 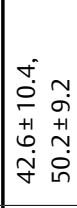 & 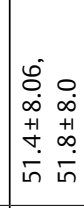 & 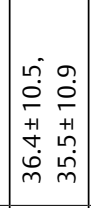 & 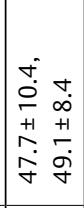 & $\begin{array}{l}0 \\
+1 \\
+1 \\
20 \\
0 \\
m \\
0 \\
0 \\
+1 \\
0 \\
0\end{array}$ & 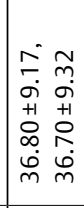 & 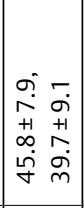 & 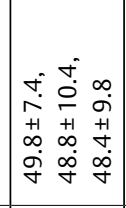 & $\begin{array}{l}\bar{F} \\
+1 \\
+1 \\
+ \\
0 \\
0 \\
+1 \\
0 \\
m\end{array}$ & 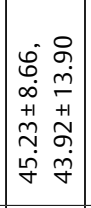 & 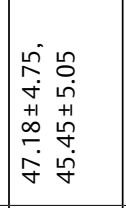 & 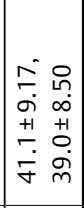 & 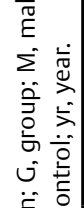 \\
\hline 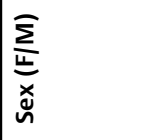 & $\begin{array}{l}\dot{N} \\
\dot{N}\end{array}$ & $\begin{array}{l}a \\
\tilde{N}\end{array}$ & $\begin{array}{l}\infty \\
\text { مீ } \\
\end{array}$ & $\begin{array}{l}\overline{2} \\
\text { i }\end{array}$ & $\frac{\sim}{z}$ & $\begin{array}{l}0 \\
\text { Dे }\end{array}$ & $\begin{array}{l}a \\
\dot{N}\end{array}$ & ¿े & a & $\stackrel{n}{\dot{n}}$ & $\begin{array}{l}0 \\
\stackrel{1}{1}\end{array}$ & $\begin{array}{l}\stackrel{\ln }{\check{m}} \\
\dot{m}\end{array}$ & 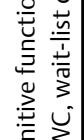 \\
\hline 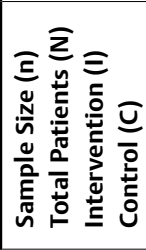 & $\begin{array}{l}\frac{m}{\pi} \\
\stackrel{n}{m} \\
\frac{m}{\pi} \\
\frac{\pi}{0} \\
\stackrel{\pi}{11} \\
z\end{array}$ & $\begin{array}{l}\stackrel{N}{\pi} \\
\ddot{u} \\
0 \\
\tilde{N} \\
\frac{\pi}{N} \\
\tilde{n} \\
z\end{array}$ & 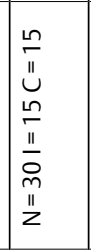 & $\begin{array}{l}\sigma \\
\bar{n} \\
u \\
\sigma \\
\frac{\pi}{11} \\
\infty \\
0 \\
11 \\
z\end{array}$ & $\begin{array}{l}0 \\
\frac{0}{11} \\
u \\
0 \\
\frac{1}{11} \\
\frac{0}{1} \\
n \\
z\end{array}$ & $\begin{array}{l}0 \\
\frac{0}{11} \\
0 \\
0 \\
\frac{11}{2} \\
\stackrel{1}{11} \\
z\end{array}$ & 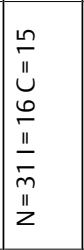 & 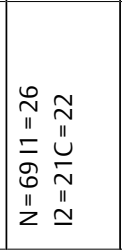 & $\begin{array}{l}m \\
\pi \\
u \\
\frac{m}{n} \\
\frac{\pi}{11} \\
\infty \\
N 1 \\
z\end{array}$ & $\begin{array}{l}\frac{m}{m} \\
u \\
m \\
\frac{\pi}{2} \\
0 \\
\pi \\
z\end{array}$ & $\begin{array}{l}\frac{\pi}{\pi} \\
u \\
\frac{\pi}{\pi} \\
\frac{\pi}{N} \\
\pi \\
z\end{array}$ & 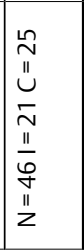 & 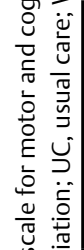 \\
\hline 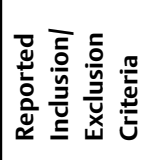 & 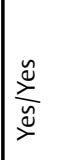 & 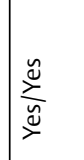 & 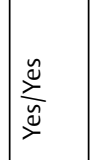 & 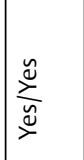 & 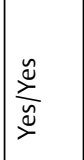 & 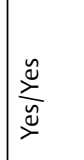 & 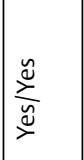 & 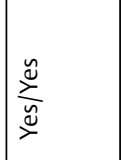 & 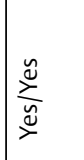 & $\frac{0}{y}$ & $\frac{0}{\frac{\partial}{y}}$ & $\sum_{\substack{y \\
$\cline { 1 - 1 }$}}$ & 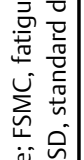 \\
\hline 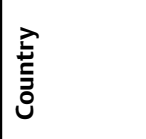 & 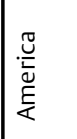 & 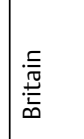 & 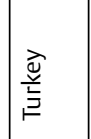 & 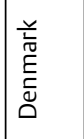 & 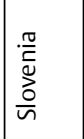 & $\underset{\mathbb{T}}{\stackrel{\complement}{\leftrightarrows}}$ & 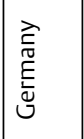 & 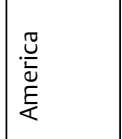 & 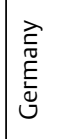 & 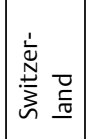 & 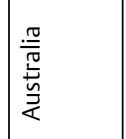 & 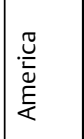 & 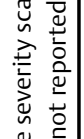 \\
\hline 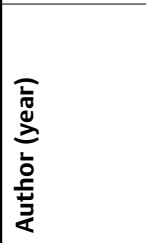 & 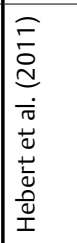 & 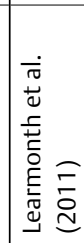 & 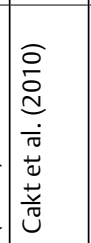 & 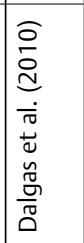 & 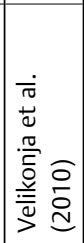 & 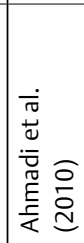 & 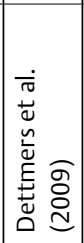 & 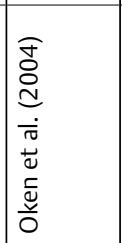 & 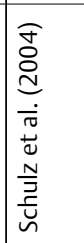 & 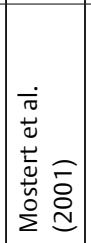 & 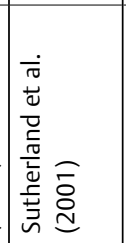 & 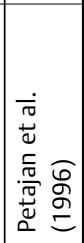 & 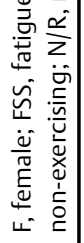 \\
\hline
\end{tabular}




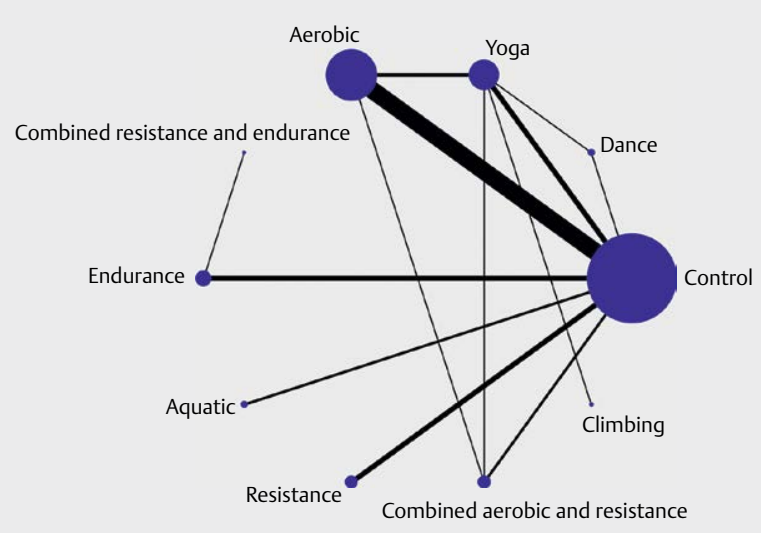

Fig. 2 Network of intervention comparisons from trials included in the network meta-analysis. The size of the nodes corresponds to the total number of patients randomized to each intervention group. The width of the lines represents the number of direct comparisons that were performed in head-to-head trials.

Switzerland [56], Turkey [57], the Netherlands [58], Belgium [59] or Slovenia [60]. Four of them were multi-arm RCTs and the rest were two-arm parallel group trials. The average age of the participants ranged from 31 to 62 , and the duration of the interventions ranged from 2 to 24 weeks. The studies included different types of exercise, such as aquatic exercise, aerobic exercise, resistance training, endurance training, dance, yoga, climbing, etc.

\section{Risk of bias and quality assessment}

The risk of bias within the studies was generally low (or probably low). Among all comparisons, random sequence generation was adequate in 21 trials (77.8\%), and allocation concealment was adequate in 17 trials (63.0\%). Blinding of outcome assessment (detection bias) was not conducted in five trials (18.5\%) [38, 48$50,55]$, and incomplete outcome data (attrition bias) was identified in four RCTs $(17.4 \%)$ [34, 36, 52, 57]. Eight trials reported an intention-to-treat analysis (36.4\%). High 'other sources of bias' was mainly due to low adherence $(<80 \%)[40,46,58]$. Notably, the limitations of the study design made it difficult for both participants and personnel to be blinded to exercise interventions, leading to a high risk of performance bias in all studies. The details of assessing the risk of bias can be found in Supplementary Figure S1.

\section{Network meta-analysis for exercise and nonpharmaceutical interventions}

Twenty-seven trials (59 arms, 1,470 participants) were included in the analysis of fatigue. The network consisted of 23 studies with two arms, 3 studies with three arms, and 1 study with four arms reporting on 10 different kinds of interventions ( 13 arms on aerobic, 5 yoga, 4 resistance training, 5 endurance training, 2 aquatic exercise, 2 combined aerobic and resistance training, 1 combined resistance and endurance training, 1 dance, 1 climbing; 25 arms on control groups). The network plot of pairwise comparisons across these trials is shown in $>$ Fig. 2.
The results of the loop-specific model ( $95 \% \mathrm{Cls}$ of all loops were truncated at zero) and Wald test $\left(X^{2}(5 d f)=6.09, p=0.298\right)$ showed no evidence of significant inconsistency (Supplementary Figure S2 and S3). In the node-splitting model, all p-values of direct and indirect comparisons were more than 0.05 . Therefore, a consistency model was used for the subsequent analysis.

The results of the network meta-analysis were detailed in - Table 2. Fig. 3a shows the outcomes presented as SMD and $95 \% \mathrm{Cl}$ for different types of exercise compared to control group.

Among these interventions, aquatic exercise ranked as the best intervention on reducing fatigue with an SMD of $-1.73(95 \% \mathrm{Cl}=$ -2.64 to -0.82). Small-to-moderate effect sizes were observed for aerobic exercise $(\mathrm{SMD}=-0.49,95 \% \mathrm{Cl}=-0.79$ to -0.20$)$, compared with the control group. Despite large or moderate effect sizes, comparisons between some interventions were not statistically significant owing to sample size constraints.

Rank probability analysis indicated that aquatic exercise had the highest probability of being ranked as the most effective intervention (SUCRA $=99.1 \%$ ), followed by aerobic exercise (SUCRA = $68.9 \%$ ), dance $($ SUCRA $=65.0 \%$ ) and resistance training (SUCRA = $54.5 \%)$. Climbing ranked as the least effective intervention (SUCRA $=11.4 \%$ ), followed by the control group (SUCRA $=22.3 \%$ ) and combined aerobic and resistance training (SUCRA $=36.3 \%$ ). The ranking probability of all included interventions is shown in - Figs. $3 a$ and 4.

\section{Subgroup analysis and sensitivity analysis}

The subgroup analysis indicated that dance ranked as the most effective fatigue reduction intervention in the subgroups of intervention duration $\geq 10$ weeks and age $\geq 45$, followed by aerobic exercise and resistance training (Supplementary Figure S4). In the sensitivity analysis to test the impact of blinding risk, the effect sizes for the 10 kinds of interventions remained almost unchanged, suggesting that the overall results were robust. Moreover, small rises were observed in the ranking probabilities of aerobic exercise, resistance training, endurance training, combined resistance and endurance training as well as combined aerobic and resistance training ( $\triangleright$ Fig. $\mathbf{3 b}$ ).

\section{Meta-regression analysis and publication bias}

To further search for the sources of heterogeneity, meta-regression analysis was conducted by publication year, sex ratio, sample size and intention to treat. The results suggested that none of these factors had significant modification effects. The shape of the funnel plot was judged and no obvious asymmetry was found, implying that there was no publication bias from small-study effects (Supplementary Figure S5).

\section{Discussion}

The current NMA is the first analysis to provide comparable evaluation of the effects of different types of exercise on reducing MSrelated fatigue. We considered 27 RCTs and compared 10 kinds of interventions, involving 1,470 patients and combining direct and indirect evidence. Several valuable findings were generated in our study. 
- Table 2 Matrix of the treatment effect estimates of all comparisons according to indirect comparison meta-analysis.

\begin{tabular}{|c|c|c|c|c|c|c|c|c|c|c|}
\hline & Aquatic & Aerobic & Dance & Resistance & Yoga & Endurance & RE & AR & Control & Climbing \\
\hline Aquatic & Aquatic & $\begin{array}{l}1.23 \\
(0.31 \\
2.16) \\
\end{array}$ & $\begin{array}{l}1.19 \\
(-0.08, \\
2.45) \\
\end{array}$ & $\begin{array}{l}1.38 \\
(0.33 \\
2.42) \\
\end{array}$ & $\begin{array}{l}1.40 \\
(0.40, \\
2.39) \\
\end{array}$ & $\begin{array}{l}1.50 \\
(0.42 \\
2.58) \\
\end{array}$ & $\begin{array}{l}1.52 \\
(0.09, \\
2.95) \\
\end{array}$ & $\begin{array}{l}1.60 \\
(0.48, \\
2.72) \\
\end{array}$ & $\begin{array}{l}1.73 \\
(0.82 \\
2.64) \\
\end{array}$ & $\begin{array}{l}2.27 \\
(0.69 \\
3.84) \\
\end{array}$ \\
\hline Aerobic & $\begin{array}{l}-1.23 \\
(-2.16 \\
-0.31)\end{array}$ & Aerobic & $\begin{array}{l}-0.05 \\
(-0.97 \\
0.88)\end{array}$ & $\begin{array}{l}0.14 \\
(-0.46, \\
0.74)\end{array}$ & $\begin{array}{l}0.16 \\
(-0.31, \\
0.64)\end{array}$ & $\begin{array}{l}0.27 \\
(-0.37 \\
0.91)\end{array}$ & $\begin{array}{l}0.28 \\
(-0.85 \\
1.42)\end{array}$ & $\begin{array}{l}0.37 \\
(-0.28 \\
1.02)\end{array}$ & $\begin{array}{l}0.49 \\
(0.20 \\
0.79)\end{array}$ & $\begin{array}{l}1.04 \\
(-0.27 \\
2.34)\end{array}$ \\
\hline Dance & $\begin{array}{l}-1.19 \\
(-2.45 \\
0.08)\end{array}$ & $\begin{array}{l}0.05 \\
(-0.88, \\
0.97)\end{array}$ & Dance & $\begin{array}{l}0.19 \\
(-0.85, \\
1.23)\end{array}$ & $\begin{array}{l}0.21 \\
(-0.68, \\
1.10)\end{array}$ & $\begin{array}{l}0.31 \\
(-0.74 \\
1.37)\end{array}$ & $\begin{array}{l}0.33 \\
(-1.08, \\
1.74)\end{array}$ & $\begin{array}{l}0.41 \\
(-0.65 \\
1.48)\end{array}$ & $\begin{array}{l}0.54 \\
(-0.35 \\
1.43)\end{array}$ & $\begin{array}{l}1.08 \\
(-0.43, \\
2.59)\end{array}$ \\
\hline Resistance & $\begin{array}{l}-1.38 \\
(-2.42 \\
-0.33)\end{array}$ & $\begin{array}{l}-0.14 \\
(-0.74 \\
0.46)\end{array}$ & $\begin{array}{l}-0.19 \\
(-1.23 \\
0.85)\end{array}$ & Resistance & $\begin{array}{l}0.02 \\
(-0.68, \\
0.72)\end{array}$ & $\begin{array}{l}0.12 \\
(-0.64, \\
0.89)\end{array}$ & $\begin{array}{l}0.14 \\
(-1.07 \\
1.35)\end{array}$ & $\begin{array}{l}0.23 \\
(-0.59 \\
1.04)\end{array}$ & $\begin{array}{l}0.35 \\
(-0.17 \\
0.87)\end{array}$ & $\begin{array}{l}0.89 \\
(-0.51, \\
2.29)\end{array}$ \\
\hline Yoga & $\begin{array}{l}-1.40 \\
(-2.39 \\
-0.40)\end{array}$ & $\begin{array}{l}-0.16 \\
(-0.64 \\
0.31)\end{array}$ & $\begin{array}{l}-0.21 \\
(-1.10, \\
0.68)\end{array}$ & $\begin{array}{l}-0.02 \\
(-0.72, \\
0.68)\end{array}$ & Yoga & $\begin{array}{l}0.10 \\
(-0.63 \\
0.83)\end{array}$ & $\begin{array}{l}0.12 \\
(-1.07 \\
1.31)\end{array}$ & $\begin{array}{l}0.21 \\
(-0.49 \\
0.90)\end{array}$ & $\begin{array}{l}0.33 \\
(-0.13 \\
0.79)\end{array}$ & $\begin{array}{l}0.87 \\
(-0.35 \\
2.09)\end{array}$ \\
\hline Endurance & $\begin{array}{l}-1.50 \\
(-2.58 \\
-0.42) \\
\end{array}$ & $\begin{array}{l}-0.27 \\
(-0.91 \\
0.37)\end{array}$ & $\begin{array}{l}-0.31 \\
(-1.37 \\
0.74)\end{array}$ & $\begin{array}{l}-0.12 \\
(-0.89 \\
0.64)\end{array}$ & $\begin{array}{l}-0.10 \\
(-0.83, \\
0.63)\end{array}$ & Endurance & $\begin{array}{l}0.02 \\
(-0.92 \\
0.95)\end{array}$ & $\begin{array}{l}0.10 \\
(-0.73 \\
0.94)\end{array}$ & $\begin{array}{l}0.23 \\
(-0.33 \\
0.79)\end{array}$ & $\begin{array}{l}0.77 \\
(-0.65 \\
2.19)\end{array}$ \\
\hline RE & $\begin{array}{l}-1.52 \\
(-2.95 \\
-0.09)\end{array}$ & $\begin{array}{l}-0.28 \\
(-1.42 \\
0.85)\end{array}$ & $\begin{array}{l}-0.33 \\
(-1.74 \\
1.08)\end{array}$ & $\begin{array}{l}-0.14 \\
(-1.35 \\
1.07)\end{array}$ & $\begin{array}{l}-0.12 \\
(-1.31 \\
1.07)\end{array}$ & $\begin{array}{l}-0.02 \\
(-0.95,0.92)\end{array}$ & RE & $\begin{array}{l}0.08 \\
(-1.17 \\
1.34)\end{array}$ & $\begin{array}{l}0.21 \\
(-0.88 \\
1.30)\end{array}$ & $\begin{array}{l}0.75 \\
(-0.95, \\
2.45)\end{array}$ \\
\hline$A R$ & $\begin{array}{l}-1.60 \\
(-2.72, \\
-0.48)\end{array}$ & $\begin{array}{l}-0.37 \\
(-1.02 \\
0.28)\end{array}$ & $\begin{array}{l}-0.41 \\
(-1.48, \\
0.65)\end{array}$ & $\begin{array}{l}-0.23 \\
(-1.04 \\
0.59)\end{array}$ & $\begin{array}{l}-0.21 \\
(-0.90, \\
0.49)\end{array}$ & $\begin{array}{l}-0.10 \\
(-0.94 \\
0.73)\end{array}$ & $\begin{array}{l}-0.08 \\
(-1.34 \\
1.17)\end{array}$ & $A R$ & $\begin{array}{l}0.13 \\
(-0.50, \\
0.75)\end{array}$ & $\begin{array}{l}0.67 \\
(-0.74 \\
2.07)\end{array}$ \\
\hline Control & $\begin{array}{l}-1.73 \\
(-2.64 \\
-0.82)\end{array}$ & $\begin{array}{l}-0.49 \\
(-0.79 \\
-0.20)\end{array}$ & $\begin{array}{l}-0.54 \\
(-1.43 \\
0.35)\end{array}$ & $\begin{array}{l}-0.35 \\
(-0.87 \\
0.17)\end{array}$ & $\begin{array}{l}-0.33 \\
(-0.79, \\
0.13)\end{array}$ & $\begin{array}{l}-0.23 \\
-0.79 \\
0.33)\end{array}$ & $\begin{array}{l}-0.21 \\
(-1.30 \\
0.88)\end{array}$ & $\begin{array}{l}-0.13 \\
(-0.75 \\
0.50)\end{array}$ & Control & $\begin{array}{l}0.54 \\
(-0.76 \\
1.84)\end{array}$ \\
\hline Climbing & $\begin{array}{l}-2.27 \\
(-3.84 \\
-0.69)\end{array}$ & $\begin{array}{l}-1.04 \\
(-2.34 \\
0.27)\end{array}$ & $\begin{array}{l}-1.08 \\
(-2.59 \\
0.43)\end{array}$ & $\begin{array}{l}-0.89 \\
(-2.29 \\
0.51)\end{array}$ & $\begin{array}{l}-0.87 \\
(-2.09 \\
0.35)\end{array}$ & $\begin{array}{l}-0.77 \\
(-2.19 \\
0.65)\end{array}$ & $\begin{array}{l}-0.75 \\
(-2.45 \\
0.95)\end{array}$ & $\begin{array}{l}-0.67 \\
(-2.07 \\
0.74)\end{array}$ & $\begin{array}{l}-0.54 \\
(-1.84 \\
0.76)\end{array}$ & Climbing \\
\hline
\end{tabular}

$A R$, aerobic and resistance; CBT, cognitive behavior therapy; ER, endurance and resistance; Reference: the i ntervention listed in the row; Data are presented as SMD and their $95 \% \mathrm{Cl}$, and a negative value indicates a better effect for the treatment written above.

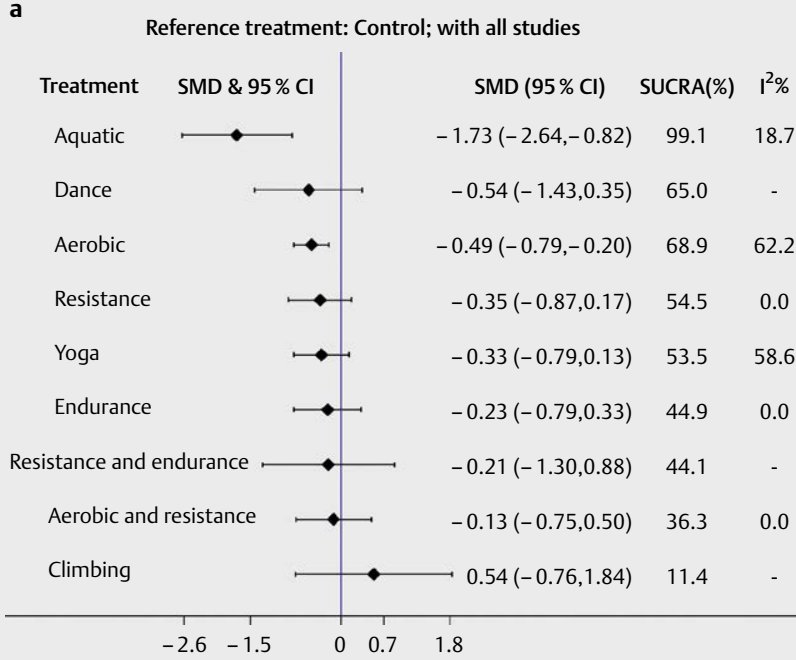

b

Reference treatment: Control; without studies with detection bias

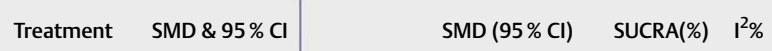

Aquatic $\longmapsto \quad-1.70(-2.63,-0.76) \quad 99.0 \quad 18.7$

Dance $\quad \longmapsto \quad \longrightarrow \quad-0.49(-1.35,0.37) \quad 64.2$

Aerobic

Resistance

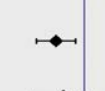

$-0.48(-0.80,-0.15) \quad 69.4 \quad 53.6$

$-0.35(-0.85,0.15) \quad 56.9 \quad 0.0$

$-0.24(-0.72,0.25) \quad 47.5 \quad 20.0$

$\begin{array}{lll}-0.22(-0.86,0.42) & 47.0 & 0.0\end{array}$

$-0.20(-1.30,0.89) \quad 45.4$

$\begin{array}{lll}-0.10(-0.71,0.50) & 36.8 & 0.0\end{array}$

Climbing

$0.64(-0.64,1.91) \quad 9.2$

- Fig. 3 Forest plots for the comparisons between the active interventions and the control intervention (a: with all studies; b: without studies with detection bias). 

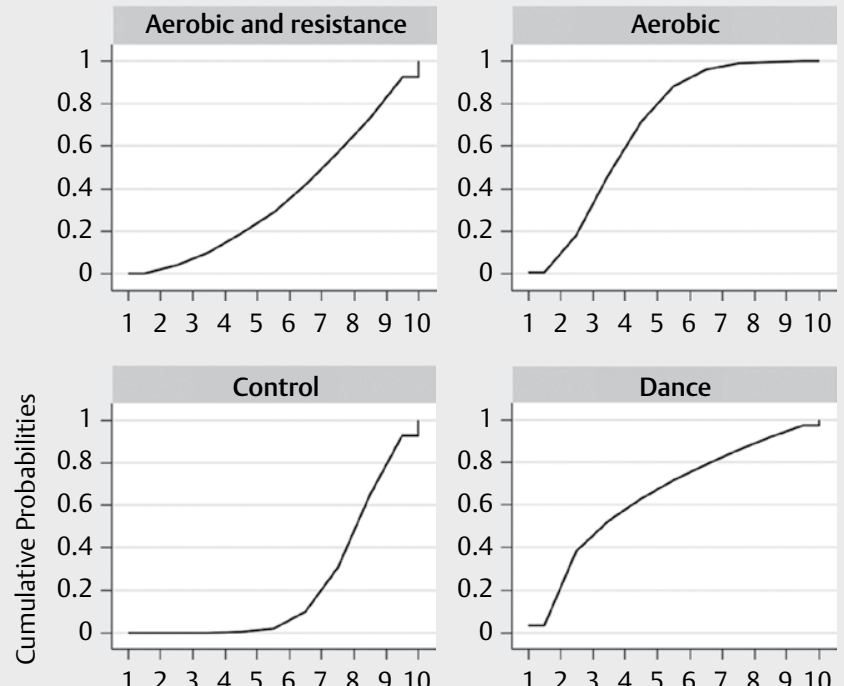

$\begin{array}{lllllllllll}1 & 2 & 3 & 4 & 5 & 6 & 7 & 8 & 9 & 10\end{array}$
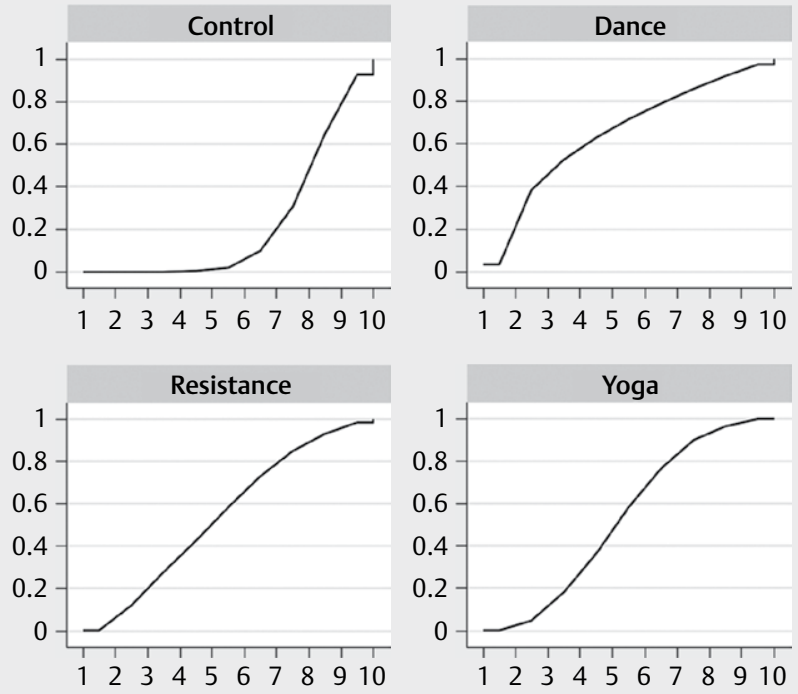
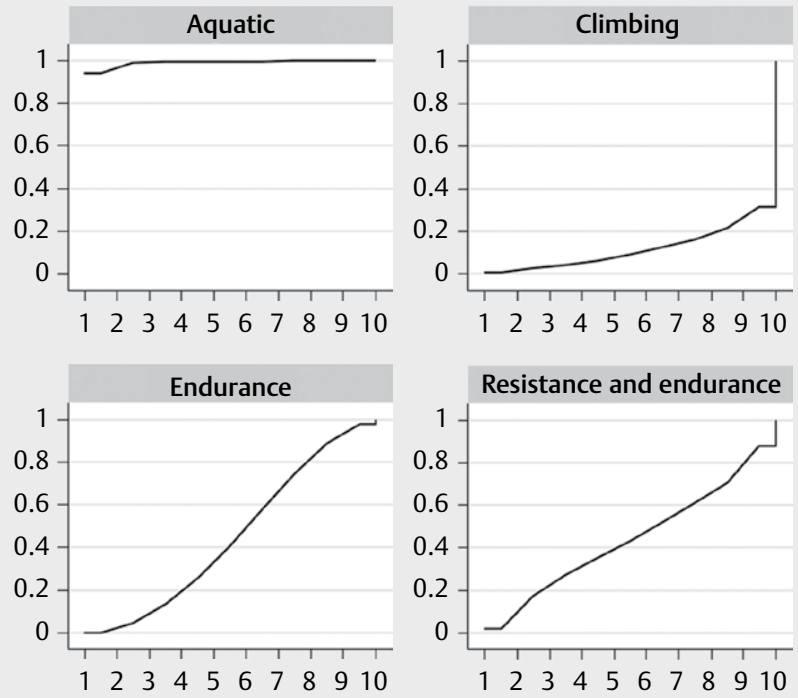

Rank

Fig. 4 Cumulative ranking probability plot for the assessment of fatigue relief in patients with MS.

According to the cumulative ranking, aquatic exercise is the most effective intervention (ranking 99.1\%) which is followed by aerobic exercise, dance, resistance training, yoga, endurance training, combined resistance and endurance training, combined aerobic and resistance training, control and climbing. These results were generally consistent with previously published traditional metaanalyses [61].

The findings emphasized the usefulness of several kinds of exercise as a valid adjunct therapy against fatigue symptoms in MS patients. Helpful and hitherto unavailable information about the comparisons between these interventions were provided. The lack of global and local inconsistency as well as the lack of small-study effects strengthened the outcomes, and the results of meta-regression and sensitivity analysis further indicated the robustness. Our ranked interventions can provide reference for future clinical trials.

The fact that aquatic exercise was more effective than other interventions may be due to the following possibilities: (a) The buoyancy, viscosity, turbulence and hydrostatical pressure of water are favorable for people with physical weakness to carry out physical activities [62]; (b) people with MS are highly sensitive to heat events, and exercise in water can reduce the temperature of body via the water temperature and prolonged training sessions [63]; and (c) water immersion can influence the activity of endogenous systems related to sodium homeostasis, including the sympathet- ic nervous system, atrial natriuretic peptide system, and renal dopa-dopamine system [64]. Aerobic exercise was observed to have small-to-moderate effect sizes in relieving fatigue in our study. Research has demonstrated the benefits of improving aerobic fitness, including reducing the risk of heart attack, lowering blood pressure, improving mental health, and improving bone mineralization [65-67]. Despite a lack of statistical differences between some comparisons, most of the interventions were shown to be better than the control group, except for climbing. Climbing was the only intervention that ranked worse than the control group, which may be correlated with the increased altitude and body temperature during climbing [68]. Our data did not support further analyses to explore these or other assumptions, and more RCTs on these topics are required in the future.

The study has several advantages. First, to our knowledge, this is the first systematic review that uses the NMA method to determine the effects and grades of a comprehensive range of exercise. Moreover, we evaluated and ranked the interventions, which can help practitioners and MS patients prioritize evidence-based interventions and then make more informed decisions.

\section{Study limitations}

Potential limitations in our NMA also merit further consideration. First, the small number of the studies for some interventions (for example, dance, climbing and aquatic exercise), limits the power 
of the results because the findings could be coincidental. Second, the interventions of the included studies were difficult to classify due to the different kinds of intervention combinations. Third, follow-up data were collected over a wide range of time intervals, from 2 to 24 weeks. The long-term effects of these interventions have not been fully elucidated and might become a focus for future research. Collectively, given the reasons above, the results must be interpreted with caution, and any association observed in the current NMA should be tested in original RCTs.

\section{Conclusion}

Although exercise has shown promising effects in relieving fatigue in MS patients, comparisons between different types of exercise are still lacking. The current indirect-comparison NMA shows the beneficial effects of aquatic exercise and aerobic training on reducing MS-related fatigue. These findings enable people with MS to choose their preferred exercise as adjunct therapy to achieve optimal management of fatigue symptoms.

\section{Conflict of Interest}

The authors declare that they have no conflict of interest.

\section{References}

[1] Noseworthy JH, Lucchinetti C, Rodriguez M et al. Multiple sclerosis. N Engl J Med 2000; 343: 938-952

[2] Browne P, Chandraratna D, Angood C et al. Atlas of Multiple Sclerosis 2013: A growing global problem with widespread inequity. Neurology 2014; 83: 1022-1024

[3] Oh J, Vidal-Jordana A, Montalban X. Multiple sclerosis: Clinical aspects. Curr Opin Neurol 2018; 31: 752-759

[4] Krupp LB, Alvarez LA, Larocca NG et al. Fatigue in multiple sclerosis. Arch Neurol 1988; 45: 435-437

[5] Sosnoff J], Socie M], Boes MK et al. Mobility, balance and falls in persons with multiple sclerosis. PLoS One 2011; 6: e28021

[6] Solaro C, Gamberini G, Masuccio FG. Depression in multiple sclerosis: epidemiology, aetiology, diagnosis and treatment. CNS Drugs 2018; 32: $117-133$

[7] Grzegorski T, Losy J. Cognitive impairment in multiple sclerosis - a review of current knowledge and recent research. Rev Neurosci 2017; 28: 845-860

[8] Patejdl R, Zettl UK. Spasticity in multiple sclerosis: Contribution of inflammation, autoimmune mediated neuronal damage and therapeutic interventions. Autoimmun Rev 2017; 16: 925-936

[9] Hoang PD, Gandevia SC, Herbert RD. Prevalence of joint contractures and muscle weakness in people with multiple sclerosis. Disabil Rehabil 2014; 36: 1588-1593

[10] Marrie RA, Cohen J, Stuve O et al. A systematic review of the incidence and prevalence of comorbidity in multiple sclerosis: Overview. Mult Scler 2015; 21: 263-281

[11] Krupp L. Fatigue is intrinsic to multiple sclerosis (MS) and is the most commonly reported symptom of the disease. Mult Scler 2006; 12: 367-368

[12] Motl RW, Mcauley E, Snook EM. Physical activity and multiple sclerosis: A meta-analysis. Mult Scler 2005; 11: 459-463
[13] Marrie RA, Horwitz RI. Emerging effects of comorbidities on multiple sclerosis. Lancet Neurol 2010; 9: 820-828

[14] Dehkordi AH. Influence of yoga and aerobics exercise on fatigue, pain and psychosocial status in patients with multiple sclerosis: A randomized trial. J Sports Med Phys Fitness 2015; 1: 219-220

[15] Motl RW, Sandroff BM. Benefits of exercise training in multiple sclerosis. Curr Neurol Neurosci Rep 2015; 15: 62

[16] Andreu-Caravaca L, Ramos-Campo DJ, Chung LH et al. Dosage and effectiveness of aerobic training on cardiorespiratory fitness, functional capacity, balance, and fatigue in people with multiple sclerosis: A systematic review and meta-analysis. Arch Phys Med Rehabil 2021; Online ahead of print. doi:10.1016/j.apmr.2021.01.078

[17] American Physical Therapy Association. Guide to Physical Therapist Practice. Part 1: A description of patient/client management. Part 2: Preferred practice patterns. Phys Ther 1997; 77: 1160-1656

[18] Cruickshank TM, Reyes AR, Ziman MR. A systematic review and meta-analysis of strength training in individuals with multiple sclerosis or Parkinson disease. Medicine (Baltimore) 2015; 94: e411

[19] Shohani M, Kazemi F, Rahmati S et al. The effect of yoga on the quality of life and fatigue in patients with multiple sclerosis: A systematic review and meta-analysis of randomized clinical trials. Complement Ther Clin Pract 2020; 39: 101087

[20] Heine M, Port Ivd, Rietberg MB et al. Exercise therapy for fatigue in multiple sclerosis. Cochrane Database Syst Rev 2015; 11: CD009956

[21] Hutton B, Salanti G, Caldwell DM et al. The PRISMA extension statement for reporting of systematic reviews incorporating network meta-analyses of health care interventions: Checklist and explanations. Ann Intern Med 2015; 162: 777-784

[22] Harriss DJ, MacSween A, Atkinson G. Ethical standards in sport and exercise science research: 2020 update. Int J Sports Med 2019; 40: 813-817

[23] Nelson ME, Rejeski W], Blair SN et al. Physical activity and public health in older adults: Recommendation from the American College of Sports Medicine and the American Heart Association. Circulation 2007; 116: 1094-1105

[24] Faigenbaum AD, Kraemer W], Blimkie C] et al. Youth resistance training: updated position statement paper from the national strength and conditioning association. J Strength Cond Res 2009; 23: S60-S79

[25] Morici G, Gruttad'Auria Cl, Baiamonte P et al. Endurance training: Is it bad for you? Breathe (Sheff) 2016; 12: 140-147

[26] Higgins JPT, Green S. Cochrane Handbook for Systematic Reviews of Interventions. Version 5.1.0. The Cochrane Collaboration, 2011. Available from www.cochrane-handbook.org.

[27] Wan X, Weng W, Liu J et al. Estimating the sample mean and standard deviation from the sample size, median, range and/or interquartile range. BMC Med Res Methodol 2014; 14: 135

[28] Cohen Jacob. A power primer. Psychol Bull 1992; 112: 155-159

[29] Lu G, Ades AE. Combination of direct and indirect evidence in mixed treatment comparisons. Stat Med 2004; 23: 3105-3124

[30] Salanti G, Ades AE, Ioannidis JPA. Graphical methods and numerical summaries for presenting results from multiple-treatment metaanalysis: an overview and tutorial. J Clin Epidemiol 2011; 64: 163-171

[31] Leucht S, Cipriani A, Spineli L et al. Comparative efficacy and tolerability of 15 antipsychotic drugs in schizophrenia: a multipletreatments meta-analysis. Lancet 2013; 382: 951-962

[32] Higgins JPT, Jackson D, Barrett JK et al. Consistency and inconsistency in network meta-analysis: Concepts and models for multi-arm studies. Res Synth Methods 2012; 3: 98-110

[33] Salanti G, Marinho V, Higgins JPT. A case study of multiple-treatments meta-analysis demonstrates that covariates should be considered. J Clin Epidemiol 2009; 62: 857-864 
[34] Raoof N, Robert M, Motahare M et al. Effect of short-term interval exercise training on fatigue, depression and fitness in normal weight vs. overweight person with multiple sclerosis. Explore (NY) 2019; 15: 134-141

[35] Kargarfard M, Shariat A, Ingle L et al. Randomized controlled trial to examine the impact of aquatic exercise training on functional capacity, balance, and perceptions of fatigue in female patients with multiple sclerosis. Arch Phys Med Rehabil 2017; 99: 234-241

[36] Kooshiar H, Moshtagh M, Sardar MA et al. Fatigue and quality of life of women with multiple sclerosis: A randomized controlled clinical trial. J Sports Med Phys Fitness 2015; 55: 668-674

[37] Ahmadi A, Arastoo AA, Nikbakht M. The effects of a treadmill training programme on balance, speed and endurance walking, fatigue and quality of life in people with multiple sclerosis. Int SportMed J 2010; 11: 389-397

[38] Ahmadi A, Arastoo AA, Nikbakht M et al. Comparison of the effect of 8 weeks aerobic and yoga training on ambulatory function, fatigue and mood status in MS patients. Iran Red Crescent Med J 2013; 15: 449-454

[39] Sadeghi Bahmani D, Razazian N, Farnia V et al. Compared to an active control condition, in persons with multiple sclerosis two different types of exercise training improved sleep and depression, but not fatigue, paresthesia, and intolerance of uncertainty. Mult Scler Relat Disord 2019; 36: 101356

[40] Young HJ, Mehta TS, Herman C et al. The effects of M2M and adapted yoga on physical and psychosocial outcomes in people with multiple sclerosis. Arch Phys Med Rehabil 2019; 100: 391-400

[41] Oken BS, Kishiyama S, Zajdel D et al. Randomized controlled trial of yoga and exercise in multiple sclerosis. Neurology 2004; 62: 2058-2064

[42] Hebert JR, Corboy JR, Manago MM et al. Effects of vestibular rehabilitation on multiple sclerosis-related fatigue and upright postural control: a randomized controlled trial. Phys Ther 2011; 91: $1166-1183$

[43] Petajan JH, Gappmaier E, White AT et al. Impact of aerobic training on fitness and quality of life in multiple sclerosis. Ann Neurol 1996; 39 : 432-441

[44] Carter A, Daley A, Humphreys L et al. Pragmatic intervention for increasing self-directed exercise behaviour and improving important health outcomes in people with multiple sclerosis: A randomised controlled trial. Mult Scler 2014; 20: 1112-1122

[45] Learmonth YC, Paul L, Miller L et al. The effects of a 12-week leisure centre-based, group exercise intervention for people moderately affected with multiple sclerosis: a randomized controlled pilot study. Clin Rehabil 2012; 26: 579-593

[46] Garrett M, Hogan N, Larkin A et al. Exercise in the community for people with minimal gait impairment due to MS: An assessor-blind randomized controlled trial. Mult Scler 2013; 19: 782-789

[47] Kerling A, Keweloh K, Tegtbur $U$ et al. Effects of a short physical exercise intervention on patients with multiple sclerosis (MS). Int J Mol Sci 2015; 16: 15761-15775

[48] Dettmers C, Sulzmann M, Ruchay-Plössl A et al. Endurance exercise improves walking distance in MS patients with fatigue. Acta Neurol Scand 2009; 120: 251-257

[49] Schulz KH, Gold SM, Witte J et al. Impact of aerobic training on immune-endocrine parameters, neurotrophic factors, quality of life and coordinative function in multiple sclerosis. J Neurol Sci 2004; 225: $11-18$

[50] Gervasoni E, Cattaneo D, Jonsdottir J. Effect of treadmill training on fatigue in multiple sclerosis: A pilot study. Int J Rehabil Res 2013; 37 : $54-60$
[51] Grazioli E, Tranchita E, Borriello G et al. The effects of concurrent resistance and aerobic exercise training on functional status in patients with multiple sclerosis. Curr Sports Med Rep 2019; 18: 452-457

[52] Dalgas U, Stenager E, Jakobsen J et al. Fatigue, mood and quality of life improve in MS patients after progressive resistance training. Mult Scler 2010; 16: 480-490

[53] Skjerbæk AG, Næsby M, Lützen K et al. Endurance training is feasible in severely disabled patients with progressive multiple sclerosis. Mult Scler 2014; 20: 627-630

[54] Dodd KJ, Taylor NF, Shields N et al. Progressive resistance training did not improve walking but can improve muscle performance, quality of life and fatigue in adults with multiple sclerosis: A randomized controlled trial. Mult Scler 2011; 17: 1362-1374

[55] Sutherland G, Andersen MB, Stoové M. Can aerobic exercise training affect health-related quality of life for people with multiple sclerosis? J Sport Exerc Psychol 2001; 23: 122-135

[56] Mostert S, Kesselring J. Effects of a short-term exercise training program on aerobic fitness, fatigue, health perception and activity level of subjects with multiple sclerosis. Mult Scler 2002; 8: 161-168

[57] Cakt BD, Nacir B, Genç H et al. Cycling progressive resistance training for people with multiple sclerosis: a randomized controlled study. Am J Phys Med Rehabil 2010; 89: 446-457

[58] Heine M, Verschuren O, Hoogervorst EL et al. Does aerobic training alleviate fatigue and improve societal participation in patients with multiple sclerosis? A randomized controlled trial. Mult Scler 2017; 23: 1517-1526

[59] Feys P, Moumdjian L, Halewyck FV et al. Effects of an individual 12-week community-located "start-to-run" program on physical capacity, walking, fatigue, cognitive function, brain volumes, and structures in persons with multiple sclerosis. Mult Scler 2019; 25: 92-103

[60] Velikonja O, Curić K, Ozura A et al. Influence of sports climbing and yoga on spasticity, cognitive function, mood and fatigue in patients with multiple sclerosis. Clin Neurol Neurosurg 2010; 112: 597-601

[61] Pilutti LA, Greenlee TA, Motl RW et al. Effects of exercise training on fatigue in multiple sclerosis: A meta-analysis. Psychosom Med 2013; 75: $575-580$

[62] Kamioka H, Tsutani K, Okuizumi H et al. Effectiveness of aquatic exercise and balneotherapy: a summary of systematic reviews based on randomized controlled trials of water immersion therapies. J Epidemiol 2010; 20: 2-12

[63] Roehrs TG, Karst GM. Effects of an aquatics exercise program on quality of life measures for individuals with progressive multiple sclerosis. J Neurol Phys Ther 2004; 28: 63-71

[64] Grossman E, Goldstein DS, Hoffman A et al. Effects of water immersion on sympathoadrenal and dopa-dopamine systems in humans. Am J Physiol 1992; 262: R993-R999

[65] Fagard RH. Physical activity, physical fitness and the incidence of hypertension. J Hypertens 2005; 23: 265-267

[66] Powell KE, Thompson PD, Caspersen C] et al. Physical activity and the incidence of coronary heart disease. Annu Rev Public Health 1987; 8: 253-287

[67] Dustman RE, Ruhling RO, Russell EM et al. Aerobic exercise training and improved neuropsychological function of older individuals. Neurobiol Aging 1984; 5: 35-42

[68] Hsieh DT, Warden GI, Butler JM et al. Multiple sclerosis exacerbation associated with high-altitude climbing exposure. Mil Med 2020; 185: e1322-e1325 